\title{
ANÁLISE DA CONDIÇÃO BUCAL DE PACIENTES PEDIÁTRICOS E ADOLESCENTES PORTADORES DE NEOPLASIAS NA INSTITUIÇÃO CASA GUIDO NA CIDADE DE CRICIÚMA (SC)
}

\section{ANALYSIS OF ORAL HEALTH OF PEDIATRIC AND TEENAGERS PATIENTS WITH NEOPLASMS IN GUIDO HOME INSTITUTION IN THE CITY OF CRICIUMA (SC)}

\author{
Maria Laura Pires Rosso* \\ Mariana Damiani Neves* \\ Paula Fernanda de Araújo*** \\ Luciane Bisognin Ceretta**** \\ Priscyla Waleska Simões"**** \\ Fernanda Guglielmi Faustini Sônego

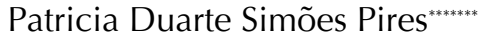

\begin{abstract}
RESUMO
A presença de patologias na cavidade oral favorece o aparecimento de alterações sistêmicas em crianças e adolescentes portadores de neoplasias e que se submetem a tratamentos agressivos, como quimioterapia e radioterapia. O objetivo deste estudo foi analisar a condição bucal dos pacientes pediátricos e adolescentes portadores de neoplasias, na instituição Casa GUIDO (Grupo pela Unidade Infanto-juvenil de Onco-hematologia) na cidade de Criciúma (SC), identificar doenças prevalentes na cavidade oral e promover ações de prevenção em saúde bucal. Foi caracterizado como um estudo epidemiológico transversal, descritivo e qualiquantitativo, totalizando uma amostra de 23 pacientes, com idade entre 2 e 16 anos, predominantemente portadores de Leucemia (39,1\%), diagnosticados entre 2008 e 2015 . Todos os indivíduos realizaram tratamento quimioterápico, sendo que 10 crianças da amostra foram submetidas a esse tratamento associado a outros métodos antineoplásicos. Através de inspeção, foram constatados 43,5\% de acometimento de cárie, 30,4\% de hiperplasia, $21,7 \%$ de mucosite e $30,4 \%$ de casos de xerostomia. Concluiu-se que alterações bucais podem ser evitadas ou minimizadas, através do desempenho do cirurgião-dentista no manejo clínico do paciente oncológico infantil.
\end{abstract}

Descritores: Neoplasias • Manifestações bucais • Prevenção • Quimioterapia

\section{A B S TRACT}

The presence of diseases in the oral cavity contributes to manifestations of systemic disorders in children and adolescents with cancer and undergoing aggressive treatments like chemotherapy and radiation. The aim of this study was to analyze the oral health of pediatric patients and adolescents with cancer in the institution Casa GUIDO (Group for Children and Teenager at Unit From Onco-hematology) in the city of Criciúma (SC), identifying prevalent diseases in the oral cavity and promoting actions prevention in oral health. It was characterized as a cross-sectional descriptive qualitative and quantitative epidemiological study, totaling a sample of 23 patients aged between 2 and 16 years, predominantly people with leukemia $(39.1 \%)$, diagnosed between 2008 and 2015. All individuals perform chemotherapy treatment, and 10 children in the sample are subjected to this treatment associated with other anticancer methods. Through inspection it was found $43.5 \%$ of carious involvement, $30.4 \%$ of hyperplasia, $21.7 \%$ of mucositis and $30.4 \%$ of cases of xerostomia. It was concluded that oral diseases can be prevented or minimized through the dentist's performance in the clinical management of cancer patient.

Descriptors: Neoplasms • Oral manifestations • Prevention • Drug therapy.

* Graduanda em Odontologia - Universidade do Extremo Sul Catarinense - Email: laura_rosso_@ @hotmail.com.

** Graduanda em Odontologia - Universidade do Extremo Sul Catarinense - Email: maridamianineves@hotmail.com.

*** Graduanda em Odontologia - Universidade do Extremo Sul Catarinense - Email: paulafernandadea@hotmail.com.

**** Doutora em Ciências da Saúde - Universidade do Extremo Sul Catarinense. Professora do Curso de Odontologia e Professora Pesquisadora no Programa de Pós-Graduação em Saúde Coletiva - Universidade do Extremo Sul Catarinense. Email: luk@unesc.net.

***** Doutora em Ciências da Saúde - Universidade do Extremo Sul Catarinense. Professora do Curso de Odontologia e Professora Pesquisadora no Programa de Pós-Graduação em Saúde Coletiva - Universidade do Extremo Sul Catarinense. Email: pri@unesc.net.

****** Mestre em odontologia - Universidade do Extremo Sul Catarinense. Professora do Curso de Odontologia - Email: fgfsonego@unesc.net

******* Doutora em Ciências da Saúde - Universidade do Extremo Sul Catarinense e professora do curso de Odontologia da Unesc - Email: patriciadspires@ gmail.com 


\section{N T R O D U Ç Ã O}

Os países desenvolvidos e em desenvolvimento enfrentam um grande problema de saúde pública: o câncer. Estatísticas mostraram que no ano de 2000 ocorreram em torno de 10 milhões de novos casos de câncer e que 6,2 milhões de pessoas morreram por essa doença ${ }^{1}$. Em relação ao câncer infantil, estatísticas apontam que uma prevalência entre $2,0 \%$ e 3,0\% dos diagnósticos correspondem a neoplasias malignas. Os tumores mais comuns relatados na literatura foram as leucemias e os linfomas ${ }^{2}$, sendo que $48,0 \%$ das crianças avaliadas foram diagnosticadas com leucemia linfoblástica aguda, seguida de $7,0 \%$ com linfoma de Burkitt, 7,0\% com neuroblastoma e $6,0 \%$ com osteossarco$\mathrm{ma}^{3}$.

Sabendo que a leucemia é o tipo de neoplasia mais comum em crianças, o $\mathrm{Ci}$ rurgião-Dentista tem grande importância no diagnóstico precoce da doença, pois as lesões primárias desse tipo de neoplasia são resultantes da infiltração de células leucêmicas nos tecidos orais, resultando em hiperplasia gengival e gengivite, e as lesões secundárias provocam aumento no sangramento gengival e susceptibilidade de infecções como candidíase e herpes simples ${ }^{4}$. Conforme um estudo apresentado, em uma amostra de 33 pacientes, antes do diagnóstico de leucemia 10 pacientes $(30,30 \%)$ apresentaram envolvimento de alterações orais devido à doença e, durante o tratamento, 27 pacientes $(81,82 \%)$ sofreram alterações na mucosa oral, principalmente presença de ulcerações, sangramento gengival e candidíase ${ }^{5}$.

O tratamento de neoplasias pode ser feito através de cirurgia, radioterapia e/ou quimioterapia e a escolha do tratamento vai depender do estágio do tumor, podendo provocar diferentes efeitos colaterais, dependendo de cada caso $^{6}$. As crianças portadoras de neoplasias relatam sequelas bucais e desconforto durante o tratamento, que podem permanecer por um longo tempo e ter consequências que podem ser potencialmente letais ${ }^{7}$. Verifica-se que $40,0 \%$ dos pacientes desenvolvem efeitos colaterais bucais após serem submetidos a quimioterapia e que, em crianças com idade inferior a 12 anos, esse número se eleva para $90,0 \%{ }^{8}$.

Entre os principais efeitos colaterais observados decorrentes das terapias antineoplásicas encontram-se a mucosite, candidíase, xerostomia, cárie de radiação, alterações no desenvolvimento crânio-facial e hemorragias ${ }^{9,10,11,12}$. Perante as complicações resultantes dos tratamentos contra o câncer e as manifestações orais decorrentes da doença, o desconforto e a dor podem ser responsáveis por provocar um déficit nutricional, alteração da resposta imunológica e um tempo maior de internação hospitalar, podendo até mesmo desencadear um processo de sepse e expor o paciente a um risco maior de vida ${ }^{9}$.

Dentro dessa perspectiva, esta pesquisa tem como objetivo analisar a condição bucal dos pacientes pediátricos e adolescentes portadores de neoplasias na instituição Grupo pela Unidade Infanto-juvenil de Onco-hematológica (Casa GUIDO) na cidade de Criciúma (SC).

\section{MÉTODOS}

Foi realizado um estudo transversal, descritivo, de abordagem quali-quantitativa, aprovado pelo Comitê de Ética do local onde foi realizada a pesquisa, sob o protocolo 986.436/2015.

A população deste estudo foi composta por crianças e adolescentes de 0 a 18 anos de idade, portadores de neoplasia e que se submeteram a terapias antineoplásicas e que participam da instituição Casa GUIDO, localizada no município de Criciúma/SC, no período de março a maio de 2015, sendo estimada uma amostra de conveniência totalizando 30 indivíduos.

Para a coleta de dados, utilizou-se um questionário que foi aplicado aos responsáveis pelos pacientes, desenvolvido pelos autores desta pesquisa, com questões relacionadas ao conhecimento sobre saúde bucal e consequências das terapias contra o câncer, além das relacionadas às questões sociodemográficas. Foi realizado, também, um exame clínico intraoral com palitos de madeira estéreis, para se observar a existência de alterações como: cárie, hiperplasia gengival, sangramento, afta,
ROSSO MLP NEVES MD ARAÚJO PF CERETTA LB

SIMÕES PW

SONEGO FGF

PIRES PDS

ANÁLISE DA

CONDIÇÃO BUCAL

DE PACIENTES

PEDIÁTRICOS E

ADOLESCENTES

PORTADORES DE

NEOPLASIAS NA

INSTITUIÇÃO

CASA GUIDO

NA CIDADE DE

CRICIÚma (SC)
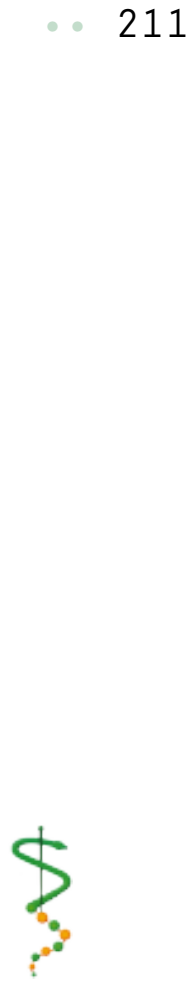

REV, ODONTOL.

Univ. CID. São

Paulo

2015; 27(3): 210-

9 , SET-DEZ 
ROSSO MLP

NEVES MD

ARAÚJO PF

CERETTA LB

SIMÕES PW

SONNEGO FGF

PIRES PDS

ANÁLISE DA CONDIÇÃO BUCAL

DE PACIENTES

PEDIÁTRICOS E

ADOLESCENTES

PORTADORES DE

NEOPLASIAS NA

INST I TU I ÇÃO

CASA GUIDO

NA CIDADE DE

CRICIÚMA (SC)

\section{2}

REV, ODONTOL.

UNIV. CID, SÃO PAULO

$2015 ; 27(3): 210-$

9 , SET-DEZ :
I SSN 1983-5183

mucosite, candidíase e sinais de xerostomia. Os questionários foram aplicados antes do exame e após o preenchimento do termo de consentimento livre e esclarecido pelos responsáveis dos indivíduos.

A variável dependente voltou-se à saúde bucal de pacientes pediátricos e adolescentes atendidos na Casa GUIDO. As independentes foram: gênero, idade, escolaridade, tipo de doença e tipo de alteração oral.

Após a coleta de dados, foi elaborado um banco de dados no software Statistical Package for the Social Sciences (SPSS) versão 22, aplicativo também utilizado para a análise estatística. Foi avaliada a normalidade de todas as variáveis numéricas pelo teste de Shapiro Wilk, que revelou distribuição normal para a idade e frequência de uso do fio dental, e não gaussiana para as demais; assim, foi calculada a média e desvio-padrão para o $1^{\circ}$ grupo, e mediana e intervalo interquartil para o $2^{\circ}$ grupo de variáveis (sem distribuição normal). Também foi estimada a frequência absoluta (n) e relativa (\%) para as variáveis qualitativas.

Foi utilizado o teste de Qui-quadrado de Pearson, sucedido da correção de continuidade de Yates (nas tabelas de con- tingência), para quantificar a associação ou independência entre a idade dicotomizada pela média e as neoplasias; as alterações e o uso de medicamentos; as alterações e uso do fio dental e frequência de escovação; e alterações e o tipo de tratamento realizado. Para todos os testes supracitados foi considerado um nível de singnificância $\alpha=0,05$ e intervalo de confiança de $95 \%$.

\section{RESULTADOS}

Inicialmente o estudo foi composto por uma amostra de 35 pacientes, porém, destes, 5 não foram localizados e 7 não tinham o diagnóstico de câncer e estavam dentro dos critérios de exclusão. A pesquisa com a amostra restante contemplou a avaliação de 23 pacientes oncológicos de 2 a 16 anos, com média de idade de 7,13 $( \pm 3,969)$, sendo que a faixa etária mais frequente foi de 3 e 6 anos, representando $17,4 \%$ do total de pacientes. Levando-se em consideração que a Casa Guido oferece assistência para crianças de diversos municípios da Região Sul, foram registradas as cidades de origem mais incidentes, sendo Criciúma e Tubarão as mais citadas, com frequência de $39,1 \%(n=9)$ e $13,0 \%$ $(n=3)$, respectivamente.

Tabela 1 - Características das neoplasias estratificadas pela Idade

Variável

Neoplasias
Leucemia
Rabdoviosarcoma
Sarcoma
Osteossarcoma
Doença de Hodgkin
Tumor de células Gigantes
Tumor Primitivo
Histiocitose
Hemofilia
Ganglioneuroblastoma
Neuroblastoma
Neurofibromatos

Idade

$\begin{array}{ll}<=\text { média } & >\text { média } \quad p \\ n(\%) & n(\%) \\ n=15 & n=8\end{array}$

$\begin{array}{lll}5(55,6 \%) & 4(44,4 \%) & \\ 1(100,0 \%) & 0(00,0 \%) & \\ 0(00,0 \%) & 1(100,0 \%) & \\ 0(00,0 \%) & 1(100,0 \%) & \\ 0(00,0 \%) & 1(100,0 \%) & \\ 1(100,0 \%) & 0(00,0 \%) & 0,258 \\ 1(100,0 \%) & 0(00,0 \%) & \\ 3(100,0 \%) & 0(00,0 \%) & \\ 1(100,0 \%) & 0(00,0 \%) & \\ 2(100,0 \%) & 0(00,0 \%) & \\ 1(100,0 \%) & 0(00,0 \%) \\ 0(00,0 \%) & 1(100,0 \%)\end{array}$


Foi verificada a presença de 12 tipos diferentes de câncer, sendo que Leucemia foi o mais recorrente, com manifestação em 9 (39,1\%) casos. As demais neoplasias estão dispostas na Tabela 1, de acordo com a faixa etária.

Os diagnósticos foram estabelecidos entre 2008 e 2015, e entende-se que a distribuição de frequência nesse período variou de acordo com a idade da criança. Em 91,3\% dos casos, os tratamentos foram iniciados no mesmo ano do diagnóstico da doença, exceto em 2 pacientes recém-nascidos $(86,6 \%)$. Todos os indiví-

Tabela 2 - Características das alterações bucais relatadas pelos pais estratificadas pelo uso de medicamentos

\begin{tabular}{|c|c|c|c|}
\hline \multirow[t]{3}{*}{ Variável } & \multicolumn{3}{|c|}{ Medicamento } \\
\hline & Sim & Não & $\mathrm{P}$ \\
\hline & $\mathrm{n}(\%)$ & $\mathrm{n}(\%)$ & \\
\hline \multicolumn{4}{|l|}{ Afta } \\
\hline Sim & $2(20,0 \%)$ & $8(80,0 \%)$ & 0,109 \\
\hline Não & $5(55,6 \%)$ & $4(44,4 \%)$ & \\
\hline \multicolumn{4}{|l|}{ Mucosite } \\
\hline Sim & $6(50,0 \%)$ & $6(50,0 \%)$ & 0,120 \\
\hline Não & $1(14,3 \%)$ & $6(85,7 \%)$ & \\
\hline \multicolumn{4}{|c|}{ Xerostomia } \\
\hline Sim & $5(38,5 \%)$ & $8(61,5 \%)$ & 0,829 \\
\hline Não & $2(33,3 \%)$ & $4(66,7 \%)$ & \\
\hline \multicolumn{4}{|l|}{ Halitose } \\
\hline Sim & $1(100,0 \%)$ & $0(00,0 \%)$ & 0,179 \\
\hline Não & $6(33,3 \%)$ & $12(66,7 \%)$ & \\
\hline \multicolumn{4}{|c|}{ Hiperplasia } \\
\hline Sim & $1(100,0 \%)$ & $0(00,0 \%)$ & 0,179 \\
\hline Não & $6(33,3 \%)$ & $12(66,7 \%)$ & \\
\hline \multicolumn{4}{|c|}{ Candidíase } \\
\hline Sim & $1(100,0 \%)$ & $0(00,0 \%)$ & 0,179 \\
\hline Não & $6(33,3 \%)$ & $12(66,7 \%)$ & \\
\hline \multicolumn{4}{|l|}{ Cárie } \\
\hline Sim & $2(20,0 \%)$ & $8(80,0 \%)$ & 0,252 \\
\hline Não & $4(44,4 \%)$ & $5(55,6 \%)$ & \\
\hline \multicolumn{4}{|c|}{ Hiperplasia } \\
\hline Sim & $2(28,6 \%)$ & $5(71,4 \%)$ & 0,829 \\
\hline Não & $4(33,3 \%)$ & $8(66,7 \%)$ & \\
\hline \multicolumn{4}{|c|}{ Sangramento } \\
\hline Sim & $3(37,5 \%)$ & $5(62,5 \%)$ & 0,636 \\
\hline Não & $3(27,3 \%)$ & $8(72,7 \%)$ & \\
\hline
\end{tabular}

ROSSO MLP NEVES MD ARAÚJO PF CERETTA LB SIMÕES PW SONEGO FGF PIRES PDS

ANÁLISE DA CONDIÇÃO BUCAL DE PACIENTES PEDIÁTRICOS E ADOLESCENTES PORTADORES DE NEOPLASIAS NA INSTITUIÇÃO CASA GUIDO NA CIDADE DE CRICIÚma (SC)

\section{3}

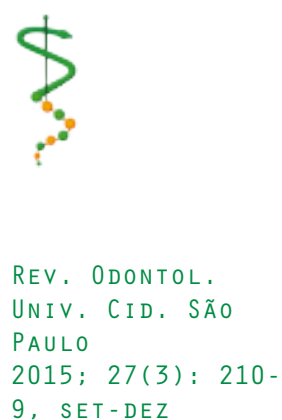


I SSN $1983-5183$

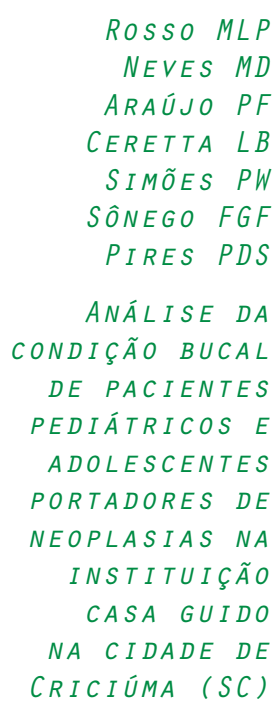

214

REV. ODONTOL.

UNIV. CID. SÃO PAULO

$2015 ; 27(3): 210-$

9 , SET-DEZ

Tabela 3 - Distribuição de frequência das patologias bucais relatadas e inspecionadas

\begin{tabular}{lll}
\hline Variável & Alterações Relatadas & Frequência \\
Quantidade de alterações & $\mathrm{n}(\%)$ & $\mathrm{n}(\%)$ \\
& $\mathrm{n}=23$ & $\mathrm{n}=23$ \\
\hline 1 alteração & $5(21,7 \%)$ & $5(21,7 \%)$ \\
Associação de 2 & $9(39,1 \%)$ & $6(26,1 \%)$ \\
Associação de 3 & $5(21,7 \%)$ & $6(26,1 \%)$ \\
Associação de 4 & - & $1(4,3 \%)$ \\
Associação de 5 & - & $1(4,3 \%)$ \\
Alteração bucal & $19(82,6 \%)$ & $19(82,6 \%)$ \\
Cárie & - & $10(43,5 \%)$ \\
Hiperplasia & $1(4,3 \%)$ & $7(30,4 \%)$ \\
Sangramento & - & $8(34,8 \%)$ \\
Afta & $10(43,5 \%)$ & $6(26,1 \%)$ \\
Mucosite & $12(52,2 \%)$ & $5(21,7 \%)$ \\
Candidíase & $1(4,3 \%)$ & $1(4,3 \%)$ \\
Xerostomia & $12(56,5 \%)$ & $7(30,4 \%)$ \\
Halitose & $1(4,3 \%)$ & - \\
\hline \hline
\end{tabular}

Fonte: dados da pesquisa

Houve 12 casos $(52,2 \%)$ de associação das neoplasias com problemas sistêmicos de saúde. Quando questionados sobre a submissão ao tratamento odontológico, 6 indivíduos responderam positivamente $(26,1 \%): 3$ casos de extração $(50 \%), 1$ caso de manutenção ortodôntica $(16,7 \%)$, 3 procedimentos restauradores $(50 \%)$ e 2 casos de profilaxia (33,3\%). É importante ressaltar que houve situações em que 2 ou mais procedimentos foram executados na mesma criança.

Considerando-se a importância da orientação quanto aos riscos da terapia antineoplásica em relação à saúde bucal, através do Médico e/ou profissional de saúde, vale salientar que 78,3\% (18) dos pacientes não receberam quaisquer informações ou alertas. Os demais responsáveis, totalizando 5 (21,7\%), foram instruídos apenas quanto ao risco de aparecimento de ulcerações leves na mucosa oral (afta).

Referente à saúde odontológica da criança, os pais foram questionados sobre a presença e/ou aparecimento de alterações bucais, bem como o tipo da lesão, e posteriormente foi executada inspeção da cavidade oral para confirmação das respostas obtidas. Também foram questionados sobre a frequência de escovação e uso de fio dental, supervisionado ou não, de modo que pudéssemos reconhecer a associação entre as lesões bucais por eles relatadas e o fluxo de limpeza da cavidade oral. Tais dados estão discriminados na Tabela 3 de distribuição de frequência e na Tabela 4 estratificada, respectivamente.

\section{I SCUS SÃO}

Atualmente, os recursos terapêuticos para o tratamento oncológico na infância estão sendo promissores, indicando que $70 \%$ das crianças acometidas por câncer podem ser curadas, quando diagnosticadas precocemente e tratadas de modo adequado em centros especializados ${ }^{13}$. A Odontologia desempenha um papel fundamental nas diferentes fases terapêuticas contra o câncer. ${ }^{14}$

Partindo desse princípio, nosso estudo objetivou analisar a condição bucal dos pacientes pediátricos e adolescentes portadores de neoplasias na instituição Casa GUIDO de Criciúma - SC, de modo que pudéssemos estabelecer índices de alterações de mucosa e do nível de instrução 
Tabela 4 - Características das alterações relatadas pelos pais, estratificadas pela escovação e uso do fio dental

\begin{tabular}{|c|c|c|c|c|c|c|c|}
\hline \multirow[b]{2}{*}{ Variável } & \multicolumn{4}{|l|}{ Escovação } & \multicolumn{3}{|c|}{ Fio Dental } \\
\hline & $\begin{array}{l}1 \mathrm{x} \\
\mathrm{n}(\%)\end{array}$ & $\begin{array}{l}2 x \\
n(\%)\end{array}$ & $\begin{array}{l}3 x \\
n(\%)\end{array}$ & $P$ & $\begin{array}{l}\text { Sim } \\
\mathrm{n}(\%)\end{array}$ & $\begin{array}{l}\text { Não } \\
\mathrm{n}(\%)\end{array}$ & $P$ \\
\hline \multicolumn{8}{|l|}{ Cárie } \\
\hline Sim & $1(10,0 \%)$ & $3(30,0 \%)$ & $6(60,0 \%)$ & 0,782 & $\begin{array}{l}2 \\
(20,0 \%)\end{array}$ & $8(80,0 \%)$ & 0,510 \\
\hline Não & $1(11,1 \%)$ & $4(44,4 \%)$ & $4(44,4 \%)$ & & $\begin{array}{l}3 \\
(33,3 \%)\end{array}$ & $6(66,7 \%)$ & \\
\hline \multicolumn{8}{|c|}{ Hiperplasia } \\
\hline Sim & $0(0,0 \%)$ & $1(100 \%)$ & $0(00,0 \%)$ & 0,179 & $0(0,0 \%)$ & $1(100 \%)$ & 0,539 \\
\hline Não & $0(0,0 \%)$ & $6(33,3 \%)$ & $12(66,7 \%)$ & & $\begin{array}{l}5 \\
(27,8 \%)\end{array}$ & $13(72,2 \%)$ & \\
\hline
\end{tabular}

ROSSO MLP

NEVES MD

ARAÚJO PF

CERETTA LB

SIMÕES PW

SONEGO FGF

PIRES PDS

ANÁLISE DA

CONDIÇÃO BUCAL

DE PACIENTES

PEDIÁTRICOS E

ADOLESCENTES

PORTADORES DE

NEOPLASIAS NA

INSTITUI ÇÃO

CASA GUIDO

NA CIDADE DE

CRICIÚMA (SC)

Sangramen-

to

Sim

$$
1(12,5 \%)
$$

$4(50,0 \%) \quad 3(37,5 \%)$

0,522

2

Não

$$
1(9,1 \%) \quad 3(27,5 \%) \quad 7(63,6 \%)
$$

$(25,0 \%)$

$6(75,0 \%)$

0,912

3

Afta

Sim

$0(0,0 \%)$

$2(20,0 \%) \quad 8(80,0 \%)$

$(27,3 \%)$

$8(72,7 \%)$

Não

$$
0(0,0 \%)
$$

$$
5(55,6 \%) \quad 4(44,4 \%)
$$

$\begin{array}{llll}0,109 & (30,0 \%) & 7(70,0 \%) & 0,701 \\ & 2 & & \\ & (22,2 \%) & 7(77,8 \%)\end{array}$

Mucosite

Sim

Não

$$
0(0,0 \%)
$$

$$
5(41,7 \%) \quad 7(58,3 \%)
$$

0,568

3

Candidíase

Sim

$$
0(0,0 \%)
$$

$2(28,6 \%) \quad 5(71,4 \%)$

\section{5}

Não

$$
0(0,0 \%) \quad 7(38,9 \%) \quad 11(61,1 \%)
$$

0,433

$0(0,0 \%) \quad 1(100 \%)$

0,539

Xerostomia

Sim

$$
0(0,0 \%)
$$

$5(38,5 \%) \quad 8(61,5 \%)$

0,829

4

Não

$$
0(0,0 \%)
$$

$2(33,3 \%) \quad 4(66,7 \%)$

$(27,8 \%) \quad 13(72,2 \%)$

$\begin{array}{ll}(41,7 \%) & 7(58,3 \%) \\ 0(0,0 \%) & 7(100 \%)\end{array}$

0,047

Halitose

Sim

$$
0(0,0 \%)
$$

$1(100 \%) \quad 0(00,0 \%)$

0,179

$6(33,3 \%) \quad 12(66,7 \%)$

$0(0,0 \%)$

Não

Fonte: dados da pesquisa

dos pais quanto à saúde bucal das crianças ${ }^{14,15}$. Acreditamos que, ao atuar na fase que antecede o tratamento radical, de forma efetiva, é possível orientar os pais e prevenir o aparecimento de complicações sistêmicas oriundas de processos infecciosos da cavidade oral.
Para crianças portadoras de neoplasias, recomenda-se que sejam examinadas pelo Cirurgião-Dentista logo que recebam o diagnóstico da doença, para que o tratamento odontológico seja feito antes de se iniciar a terapia antineoplásica, o que confirma a importância desse profissional
REV, ODONTOL. UNIV, CID, SÃo PAULO

$2015 ; 27(3): 210-$ 9 , SET-DEZ 
ROSSO MLP

NEVES MD

ARAÚJO PF

CERETTA LB

SIMÕES PW

SONNEGO FGF

PIRES PDS

ANÁLISE DA CONDIÇÃO BUCAL

DE PACIENTES

PEDIÁTRICOS E

ADOLESCENTES

PORTADORES DE

NEOPLASIAS NA

INST I TU I ÇÃO

CASA GUIDO

NA CIDADE DE

CRICIÚMA (SC)

\section{6}

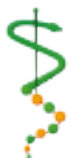

REV, ODONTOL.

UNIV, C I D , S ÃO

PAULO

$2015 ; 27(3): 210-$

9, SET-DEZ como integrante de uma equipe interdisciplinar no tratamento do câncer ${ }^{16,17,18}$.

Nosso estudo contemplou a avaliação de 23 pacientes, entre 2 e 16 anos, com 11 neoplasias distintas e apenas $26,1 \%$ da amostra havia se submetido a algum procedimento odontológico. Fato este que se opõe à literatura, quando afirma que uma avaliação minuciosa, por parte do Cirurgião-Dentista, deve ser executada antes de se iniciar o tratamento oncológico, através de métodos radiográficos, exame clínico e anamnese completa ${ }^{19}$. Da mesma forma, diversos estudos expõem que a adequação do meio bucal, que antecede a terapia antineoplásica, deve se dar por meio da eliminação de fontes de trauma, restaurações de dentes ou fraturas; extração de dentes decíduos que se encontram no período de esfoliação; se necessário realizar aplicação de selantes como medida preventiva contra a cárie; realizar os tratamentos endodônticos e exodontias quando indicados como medidas para diminuir possíveis complicações ${ }^{13,15,19}$.

O tratamento preventivo também se estende às orientações relacionadas ao controle da dieta, evitando-se alimentos ásperos, espessos, condimentados, picantes, sucos cítricos e bebidas alcoólicas que possam causar irritação ou queimadura na mucosa bucal, devendo-se dar preferência para os alimentos frios e líquidos que desencadeiam uma sensação de alívio ${ }^{8,20}$.

Um estudo com uma equipe de enfermagem, pacientes e acompanhantes do setor oncológico no Hospital Infantil Joana de Gusmão na cidade de Florianópolis (SC) aponta em 78,9\% dos questionários aplicados o relato da inexistência de um protocolo de autocuidado em saúde bucal ao paciente hospitalizado ${ }^{3}$. Tal informação corrobora nossos resultados, em que $78,3 \%$ dos pesquisados responderam de forma negativa quando questionados se haviam recebido instrução profissional sobre higiene bucal ou sobre os riscos de aparecimento de manifestações bucais em decorrência do tratamento antineoplásico.

Entre as complicações orais oriundas da terapia contra o câncer, a mucosite é destacada como a lesão de maior frequência e o maior fator dose-limitante para o tratamento radioterápico na região de cabeça e pescoço ${ }^{21}$. De acordo com um estudo, não apenas pelo uso de radiação, a mucosite, em associação ou não com outras lesões, foi a mais recorrente em ambos os sexos e em todas as idades e acomete cerca de $40 \%$ dos pacientes pediátricos que são submetidos à quimioterapia ${ }^{22}$. Nossos registros vão de encontro a tais afirmações, visto que a lesão supracitada não foi a mais incidente, ficando em $5^{\circ}$ lugar com taxa de $21,7 \%$, apresentando associação estatisticamente significativa $(p=0,047)$ relacionada ao uso de fio dental.

Para fins de esclarecimento, vale ressaltar que a mucosite é caracterizada pela inflamação, ulceração da mucosa oral e o recobrimento da mucosa por uma membrana fibrino-purulenta. Os sintomas mais comuns são a falta de apetite, disfagia e dor intensa que pode levar à desnutrição, maior susceptibilidade a infecções sistêmicas ou orais e comprometimento da higiene oral ${ }^{6,7,8,20,23,24,25,26,27}$.

Outra lesão encontrada em $4,3 \%$ dos casos foi a candidíase, considerada a infecção oportunista fúngica mais comum na cavidade oral e envolve frequentemente os tecidos moles do lábio, a mucosa bucal, a língua, o palato e a mucosa faríngea ${ }^{6,7}$. A sepse fúngica representa a causa de um número significante de casos de mortalidade de pacientes com câncer, sendo que $60 \%$ dos casos têm associação com a infecção primária bucal ${ }^{6}$.

Diversos fatores contribuem para o desenvolvimento de processos infecciosos pela sp. Candida, como a mielossupressão, comprometimento do fluxo salivar, injúrias na mucosa e lesões de mucosi$\mathrm{te}^{28}$. Dessa forma, podemos concluir que o caso de candidíase reportado em nossa pesquisa estava associado a outras lesões orais, visto que $60,8 \%$ da amostra total apresentavam uma ou mais complicações concomitantemente.

A xerostomia, prevalente em 30,4\% dos casos avaliados em nossa pesquisa, é uma condição clínica caracterizada pela disfunção das glândulas salivares, levando à redução ou ausência do fluxo salivar, podendo aumentar o risco de infecções ${ }^{25}$. A radiação tem o potencial risco de afetar as glândulas salivares e, quando isso ocorre, o fluxo de saliva pode diminuir em até 
$90,0 \%$, sendo que a saliva residual pode tornar-se viscosa, apresentando déficit nas suas funções de lubrificação e proteção ${ }^{23}$.

A etiologia da xerostomia está vinculada à diminuição da capacidade tampão da saliva (aumentando os níveis de desmineralização) e da quantidade de mucina (causando desidratação e dificuldade de proteção), assim interferindo nas propriedades de regulação microbiológica do organismo e, consequentemente, da cavidade oral ${ }^{29}$. A diminuição do fluxo salivar predispõe o aparecimento de diversas lesões orais de diferentes gravidades como: dificuldade de falar e se relacionar devido à xerostomia (boca seca), a halitose, a cárie e a dor ${ }^{30}$.

Nesse segmento, as cáries por radiação caracterizam-se por apresentarem-se localizadas nas margens cervicais dos dentes e surgem geralmente entre dois meses e um ano após o início do tratamento radioterápico ${ }^{31}$. Devido ao fato de que a cárie foi a lesão verificada com o maior índice em nossas análises (43,5\%), podemos inferir que a xerostomia pode levar a uma mudança no paladar do paciente, levando este a modificar a sua dieta, que passa a ser composta por alimentos macios e mais cariogênicos. Nesse contexto, o paciente encontra dificuldades na realização da higiene oral e apresenta sensibilidade dental, de forma que uma janela de infectividade se abre e favorece o desenvolvimento de lesões cariosas ${ }^{19,32}$.

Referente à intervenção simultânea de métodos antineoplásicos, verificamos que $43,5 \%$ dos pacientes realizaram 2 ou mais tratamentos, sendo que a quimioterapia era incidente em todos $(100 \%)$. Desse modo, Scully e Porter ${ }^{33}$ (2000), Minicucci et al. ${ }^{34}$ (2003) e Campos et al. ${ }^{35}$ (2004) nos alertam de que a radioterapia e a quimioterapia também podem ser responsáveis por alterar o processo de odontogênese, hipoplasia de esmalte, ausência de formação dentária, microdontias, alterações na rizogênese e alterações na câmara pulpar e o mais importante é que os efeitos das complicações orais podem ser agravados quando as duas terapias são associadas $33,34,35$.
Ainda que todas as crianças avaliadas tenham relatado executar a escovação pelo menos uma vez ao dia, nos preocupa o fato de que essa higiene não foi orientada por um profissional, e nem sempre é monitorada por um responsável. Essas informações nos mostram que as orientações sobre a saúde oral são essenciais no período de internação hospitalar da criança, bem como nas visitas odontológicas ${ }^{36}$. O profissional precisa estar apto a promover a saúde oral dos pacientes, estimular estes e suas famílias a enfrentarem todas as etapas que vão desde o diagnóstico, passando pela fase do tratamento até $\mathrm{O}$ acompanhamento final, com o objetivo de devolver à criança a sua saúde integral $^{13}$.

\section{CONCLUSÕES}

O perfil predominante na amostra foram pacientes com idade de 3 e 6 anos, sendo a Leucemia o tipo de câncer mais recorrente. Em decorrência dos tratamentos antineoplásicos, várias complicações na cavidade bucal são passíveis de serem observadas, entre elas mucosite, xerostomia, hemorragias gengivais, infecções dentárias ou oportunistas, alterações no paladar, distúrbios na formação dos germes dentários, cárie de radiação e osteorradionecrose, entre outras. Essas alterações bucais podem ser evitadas ou principalmente minimizadas, através do desempenho da equipe multidisciplinar no manejo clínico do paciente oncológico infantil. Nosso estudo apresentou alguns vieses em relação ao tamanho limitado da amostra e à dificuldade de localizar todos os pacientes, tendo-se em vista que estes não têm um dia fixo para frequentar a instituição e muitos permanecem internados. A qualidade de vida é possível de melhorar tanto durante quanto após as terapias antineoplásicas. Consideramos essencial a inserção do Cirurgião-Dentista como parte integrante de uma equipe multidisciplinar no atendimento de pacientes em tratamentos antineoplásicos, uma vez que a sua efetiva atuação na prevenção de doenças da cavidade oral é uma valiosa contribuição ao bem-estar desses pacientes.
ROSSO MLP NEVES MD ARAÚJO PF CERETTA LB SIMÕES PW SONNEGO FGF PIRES PDS

ANÁLISE DA CONDIÇÃO BUCAL DE PACIENTES PEDIÁTRICOS E ADOLESCENTES PORTADORES DE NEOPLASIAS NA INSTITUIÇÃO CASA GUIDO NA CIDADE DE CRICIÚMA (SC)
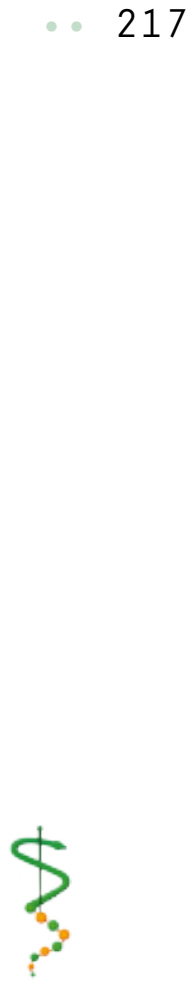

REV, ODONTOL,

UnIV. CID, SÃO PAULO

$2015 ; 27(3): 210-$

9, SET-DEZ 
ROSSO MLP

NEVES MD

ARAÚJO PF

CERETTA LB

SIMÕES PW

SONNEGO FGF

PIRES PDS

ANÁLISE DA CONDIÇÃO BUCAL

DE PACIENTES

PEDIÁTRICOS E

ADOLESCENTES

PORTADORES DE

NEOPLASIAS NA

INST I TU I ÇÃO

CASA GUIDO

NA CIDADE DE

CRICIÚMA (SC)

\section{8}

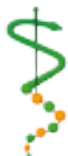

REV, ODONTOL.

UNIV, C I D , S ÃO PAULO

$2015 ; 27(3): 210-$

9, SET-DEZ
1. Brasil. Instituto Nacional de Câncer. Câncer no Brasil: dados dos registros de base populacional. Rio de Janeiro: INCA; 2003.

2. Brasil. Instituto Nacional de Câncer. Câncer na criança e adolescente no Brasil: dados dos registros de base populacional e mortalidade. Rio de Janeiro: INCA; 2008.

3. Barbosa AM, Ribeiro DM, Caldo-Teixeira AS. Conhecimentos e práticas em saúde bucal com crianças hospitalizadas com câncer. Ciênc saúde coletiva. 2010 jun; 15(supl.1):1113-22.

4. Collard MM, Hunter ML. Dental care in acute lymphoblastic leukaemia: experiences of children and attitudes of parents. Int J Paediatr Dent. 2001 Jul; 11 (4): 274-80.

5. Santos VI, Anbinder AL, Cavalcante ASR. Leucemia no paciente pediátrico: atuação odontológica. Cienc Odontol Bras. 2003; 6(2): 49-57.

6. Childers NK, Stinnett EA, Wheeler P, Wright JT, Castleberry RP, Dasanayake AP. Oral complications in children with cancer. Oral Surg Oral Med Oral Pathol Oral Radiol. 1993 Jan; 75(1): 41-7.

7. Chin, EA. A brief overview of the oral complications in pediatric oncology patients and suggested management strategies. J Dent Child. 1998; 65(6): 468.

8. Sonis ST, Fazio RC, Fang L. Complicações bucais da quimioterapia do câncer: princípios e prática de medicina oral. 2.ed. São Paulo: Guanabara Koogan; 1995.

9. Ferretti GA, Raybould TP, Brown AT, Macdonald JS, Greenwood M, Maruyama Y, Geil J, Lillich TT, Ash RC. Chlorhexidine prophylaxis for chemotherapy and radiotherapy-inducestomatitis: a randomized double-blindtrial. Oral Surg Oral Med Oral Pathol. 1990 Mar; 69(3): 331-8.
10. Minicucci EM, Dib LL, Curi MM, Shinohara EH, Sêneda LM. Sequelas odontológicas do tratamento rádio e quimioterápico em crianças. Rev Paul Pediatr. 1994 jul; 12(2): 258-63.

11. Rey E, Michelet MDC. Tratamiento de las complicaciones bucales en pacientes oncohematológicos, durante la granulocitopenía producida por la quimioterapía. Rev Fac Odontol (B.Aires). 1994; 14(35): 70-6.

12. Fonseca MA. Pediatric bone marrow transplantation: oral complications and recommendations for care. Pediatr Dent. 1998 Nov-Dec; 20(7): 386-94.

13. Kroetz FM, Czlusniak GD. Alterações bucais e condutas terapêuticas em pacientes infanto-juvenis submetidos a tratamentos anti-neoplásicos. Publ UEPG Ci Biol Saúde, Ponta Grossa. 2004 jun; 9(2): 41-8.

14. Cardoso MFA, Novikoff S, Tresso A, Segreto RA, Cervantes O. Prevenção e controle das sequelas bucais em pacientes irradiados por tumores de cabeça e pescoço. Radiol. 2005 abr; 38(2): 107-15.

15. Camargo JDFB, Dalcin Fl, Ferreira SLM. Complicações bucais imediatas do tratamento oncológico infantil: identificação, prevenção e tratamento. JBP- Rev Ibero-am Odontopediatr Odontol Bebê, 2004; 7(36): 177.

16. Albuquerque RA, Morais VLL, Sobral APV. Protocolo de atendimento odontológico a pacientes oncológicos pediátricos: revisão de literatura. $\operatorname{Rev}$ Odontol UNESP. 2007; 36(3): 275-80.

17. Osterne RLV, Brito RGM, Nogueira RLM, Soares ECS, Alves APNN, Moura JFB et al. Saúde bucal em pacientes portadores de neoplasias malignas: estudo clínico-epidemiológico e análise de necessidades odontológicas de 421 pacientes. Rev Bras Cancerol. 2008; 54(3): 221-6. 
18. Frazão COB, Alfaya TA, Costa RC, Rocha ML, Gouvêa CVD, Morais AP. Pacientes oncológicos pediátricos: manifestações bucais da terapia antineoplásica. Rev. Saúde Pesq.; 2012; 5(3): 587-92.

19. Camargo AM. O tratamento pediátrico oncológico, suas complicações orofaciais e prevenção [Monografia de Especialização]. São Paulo: Associação Brasileira de Ensino Odontológico; 2001.

20. Ingraci-de Lucia MB, Lopes-Neto FC, Padovani-Junior JÁ, Branchini PS, Nonatos ER. Protocolo de abordagem terapêutica para mucosite radio induzida. Rev bras patol oral. 2004 out-dez; 3(4): 208-10.

21. Vieira ACF, Lopes FF. Mucosite oral: efeito adverso da terapia antineoplásica. Rev Cien Méd Biol. 2006 dez; 5(3): 268-74.

22. Hespanhol FL, Tinoco EMB, Teixeira HGC, Falabella MEV, Assis NMSP. Manifestações bucais em pacientes submetidos à quimioterapia. Ciênc saúde coletiva 2010 jun; 15( Suppl 1 ): 1085-94.

23. Lopes MA, Coletta RD, Alves FA, Abbade N, Rossi Junior A. Reconhecendo e controlando os efeitos colaterais da radioterapia. Rev Assoc Paul Cir Dent. 1998 maio-jun; 52(3): 241-4.

24. Dumbrigue HB, Sandow PL, Nguyen $\mathrm{KH}$, Humphreys-Beher MG. Salivary epidermal growth factor levels decrease in patients receiving radiation therapy to the head and neck. Oral Surg Oral Med Oral Pathol Oral Radiol Endod. 2000 Jun; 89(6):710-6.

25. Massler CF Jr. Preventing and treating the oral complications of cancer therapy. Gen Dent. 2000 Nov-Dec; 48(6): 652-5.

26. Martins ACM, Caçador NP, Gaeti WP. Complicações bucais da quimioterapia antineoplásica. Acta Scientiarum 2002; 24(3): 663-70.
27. Neville B, Damm, DD, Allen, CM. Patologia oral e maxilofacial. 2.ed. Rio de Janeiro: Guanabara Koogan, 2004.

28. Gordón-Nú ezz MA, Pinto LP. Candidíase e sua relação com a mucosite oral em pacientes oncológicos pediátricos. Rev Bras Patol Oral; 2003; 2(2): 4-9.

29. Coelho CMP Sousa YTCS, Daré AMZ, Pereira ACCl, Cardoso CM. Implicações clínicas da xerostomia: abordagens sobre o diagnóstico e tratamento. Rev Assoc Paul Cir Dent; 2002 jul-ago; 56(4): 295-8.

30. Feio M, Sapeta P. Xerostomia em cuidados paliativos. Acta Med Port; 2005; 18(1): 459-66.

31. Fayle SA, Duggal MS, Williams SA. Oral problems and the dentist's role in the management of pediatric oncology patients. Dent Update. 1992 May;19(4): 152-6, 158-9.

32. Matos IS, Silva IL, Agra CM, Souza MM. Avaliação do fluxo salivar em pacientes submetidos a radioterapia de cabeça e pescoço. Rev Fac Odontol Univ Fed Bahia. 1994-1995 jan-dez; 14(15): 6-10.

33. Scully C, Porter S. ABC of oral health. Swellings and red, white, and pigmented lesions. BMJ. 2000 Jul 22; 321(7255): 225-8.

34. Minicucci EM, Lopes LF, Crocci AJ. Dental abnormalities in children after chemotherapy treatment for acute lymphoid leukemia. Leuk Res. 2003 Jan; 27(1): 45-50.

35. Campos VF, Vasconcelos FAF, Prado EFG, Cristófani LM, Vizeu HWM. Alterações no desenvolvimento dentofacial em pacientes da oncopediatria. JBC jbras clin odontol integr; 2004 mar-abr; 8(44): 101-4.

36. Altamirano EHD, Jereissati L. A fisioterapia respiratória e o processo de hospitalização criança-mãe. Psicologia: teoria e prática, 2002, 4(2): 57-65.

Recebido em 12/01/2016

Aceito em 08/03/2016
ROSSO MLP NEVES MD ARAÚJO PF

CERETTA LB

SIMÕES PW

SONEGO FGF

PIRES PDS

ANÁLISE DA

CONDIÇÃO BUCAL DE PACIENTES PEDIÁTRICOS E ADOLESCENTES PORTADORES DE NEOPLASIAS NA INSTITUIÇÃO CASA GUIDO NA CIDADE DE CRICIÚma (SC)

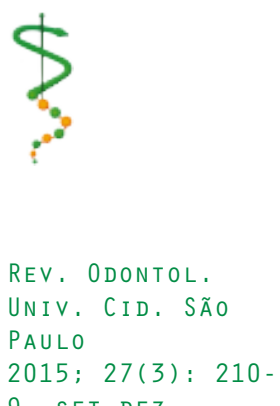

9 , SET-DEZ 